\title{
Retroperitoneal Leiomyosarcoma with Left Hip Pain as its Clinical Manifestation: A Case Report and Literature Review
}

\section{Yunfu Shi}

Tongde Hospital Of Zhejiang Province https://orcid.org/0000-0001-7532-8738

\section{Bing Zhang}

Tongde Hospital Of Zhejiang Province

\section{Mingxia Zhang}

Tongde Hospital Of Zhejiang Province

\section{Wenjuan Yang}

Tongde Hospital Of Zhejiang Province

\section{Yiming Qi}

Tongde Hospital Of Zhejiang Province

\section{Xiufang Hong ( $\triangle$ 952420845@qq.com )}

Zhejiang Hospital https://orcid.org/0000-0002-9789-982X

\section{Case report}

Keywords: Retroperitoneal tumor, leiomyosarcoma, hip pain, surgical treatment, case report

Posted Date: January 19th, 2021

DOl: https://doi.org/10.21203/rs.3.rs-146997/v1

License: (9) (i) This work is licensed under a Creative Commons Attribution 4.0 International License. Read Full License 


\section{Abstract}

Background: Leiomyosarcoma is a malignant tumor that originates from smooth muscle tissue, and can occur in multiple organs and tissues of the abdominal cavity, and most often in the retroperitoneum. It can occur at any age, usually 40 to 70 years old, and it is more common in women. The main clinical manifestations were abdominal discomfort, abdominal mass, abdominal pain, weight loss, nausea, vomiting or edema of the lower limbs. It is easy to invasion, recurrence and metastasis, and its prognosis is poor. Surgical resection is the preferred treatment method.

Case presentation: A 55-year-old woman had pain in the left hip more than 6 years. Abdominal enhanced $\mathrm{CT}$ and MR showed that the tumor was located on the left side of psoas major muscle. She underwent surgical treatment, and the postoperative pathology was leiomyosarcoma. No other treatment was performed after the operation. The patient has been followed up for 78 months and no tumor recurrence.

Conclusions: Retroperitoneal leiomyosarcoma is a rare type of malignant tumor with no specific clinical manifestation. Because of its atypical clinical manifestations, particular attention should be paid to avoid misdiagnosis and missed diagnosis.

\section{Background}

Retroperitoneal sarcoma is a rare type of malignant tumor, accounting for about $15 \%$ of soft tissue sarcoma ${ }^{1}$, in which retroperitoneal leiomyosarcoma is only $15 \sim 30 \%$ of retroperitoneal sarcoma ${ }^{2-4}$. Its etiology is still unclear. It is easy to invasion, recurrence and metastasis, and its prognosis is poor. It is the tumor with the lowest survival rate among all soft tissue sarcoma ${ }^{5}$. Retroperitoneal leiomyosarcoma is clinically rare, and cases with left hip pain as clinical manifestations are even rarer. This paper reported the diagnosis and treatment of a case of retroperitoneal leiomyosarcoma with left hip pain as the main clinical manifestation, and reviewed the relevant literature to provide references for the diagnosis and treatment of retroperitoneal leiomyosarcoma.

\section{Case Presentation}

A 55-year-old woman with a 6-year history of left hip pain was admitted to our hospital in April 2014. Medical history review: The patient had pain in the left hip without any obvious cause more than 6 years ago. The pain which seriously affected her quality of life was fixed on the left hip and radiated to the front of the pelvic cavity. The patient had been admitted to several hospitals. CT of the lumbar spine showed degeneration of the sacroiliac joints on both sides, and no obvious abnormalities in the bone ECT. Neither oral analgesia nor relaxation of skeletal muscle was effective. In February 2014, due to the aggravation of pain, the patient was admitted to our hospital for further treatment. Physical examination revealed an egg-sized mass can be touched in the left abdomen with hard texture and clear boarders when the elbow knee position was taken. Abdominal enhanced CT and MR showed that the tumor was located on the left side of psoas major muscle (Fig. 1,2). Retroperitoneal malignant tumors was 
considered, sarcoma be considered first. Retroperitoneal mass resection was performed in May 2014. Postoperative pathology: (retroperitoneal) malignant mesenchymal tumor (Fig. 3). Immunohistochemical: SMA(+), Des(+), CD34(-), CD117(-), S100(-), VIM(+), P53(+), Ki-67(+,30\%), DOG1(-) (Fig. 4). Combined with immunohistochemical results, leiomyosarcoma was diagnosed. No other treatment was performed after the operation. After 3 months of follow-up, the pain of left hip disappeared. So far, no tumor recurrence had been found, and the patient was still under follow-up.

\section{Disscussion}

Leiomyosarcoma is a malignant tumor that originates from smooth muscle tissue, and can occur in multiple organs and tissues of the abdominal cavity, and most often in the retroperitoneum. The tumors are mostly located on the upper left retroperitoneum, but rarely occur on the right side. The pelvic floor is the second most common site. It can occur at any age, usually 40 to 70 years old, and it is more common in women. This case was a middle-aged woman. The tumor was located on the left side of psoas major muscle, which was consistent with the previous literature reports. Retroperitoneal leiomyosarcoma lacks specific symptoms due to its deep anatomical location, large space and insidious onset. The main clinical manifestations were abdominal discomfort, abdominal mass, abdominal pain, weight loss, nausea, vomiting or edema of the lower limbs, however, cases with left hip pain as the clinical manifestation have not been reported. The clinical manifestations of this patient was special, with left hip pain as the primary symptom, which may be the cause of missed diagnosis.

Imaging examination is an important method for the diagnosis of retroperitoneal leiomyosarcoma. CT is the most important imaging method to evaluate the extent of tumor and the invasion of surrounding structures. Retroperitoneal leiomyosarcoma is usually large, the mass is heterogeneous, and the center may be low-density, suggesting hemorrhage, necrosis, or cystic degeneration. The edge of the primary tumor or metastasis may show moderate enhancement. Smaller masses are generally homogeneous. Liver metastases are mostly accompanied by central necrosis. Although calcification is not common, it has been reported ${ }^{6}$. MRI examination is more accurate for tumor positioning and judging its involvement in surrounding structures, especially when evaluating tumor boundaries, blood vessel abundance and blood vessel invasion ${ }^{7}$. The value of PET-CT examination has not been confirmed, but it can be used as an option when determining whether there is recurrence or metastasis. The CT and MR findings of this patient were consistent with those reported in the literature.

Pathological diagnosis is the gold standard for the diagnosis of retroperitoneal leiomyosarcoma. Retroperitoneal leiomyosarcoma shows invasive growth and tumor necrosis was common. Its histological characteristics are that the spindle-shaped tumor cells are arranged in a weave and bundle, mitoses are more common, and nuclear atypia is obvious. Combining tumor size, heteromorphism, whether it is accompanied by necrosis and mitosis and other indicators help to establish the histological grade of the tumor, of which mitosis is the most reliable indicator ${ }^{8}$. Morphological features are the most important basis for the diagnosis of leiomyosarcoma. Sometimes it is necessary to make diagnosis and differentiation combined with immunohistochemistry. Immunohistochemistry of leiomyosarcoma often 
expressed myogenic markers such as smooth muscle actin SMA and binding protein Des, while CD117 and CD34 are negatively expressed. The cell morphology, mitotic figures and immunohistochemical expression of this patient were consistent with the characteristics of leiomyosarcoma.

So far, surgery is still the main treatment for retroperitoneal leiomyosarcoma. However, the tumor volume is often large and closely related to the surrounding important blood vessels and organs, which affects surgical resection. Even after complete resection of the primary tumor, $50 \%$ of the patients may recur, and the resection of recurred tumor may still benefit. Lang ${ }^{9}$ reported that resection of liver metastases can prolong the survival time of patients. The role of radiotherapy in radical treatment of leiomyosarcoma is very limited, but it still has palliative effect in some patients ${ }^{10}$. An analysis of 261 cases of retroperitoneal soft tissue sarcoma suggested that postoperative adjuvant radiotherapy could reduce the 5-year local recurrence rate from $36-21 \%{ }^{11}$. leiomyosarcoma is not sensitive to chemotherapy, and the effect of postoperative adjuvant chemotherapy is still uncertain. For locally advanced or metastatic tumors, chemotherapy, although incurable, may delay tumor progression. Doxorubicin, epirubicin, liposome doxorubicin, isocyclophosphamide or anthracycline-containing combination regimen are commonly used palliative chemotherapy regimens. Trabectedin is approved for use in patients with advanced soft tissue sarcoma who have failed the treatment of anthracyclines and ifosfamide or are not suitable for treatment with these drugs, and has a good effect on leiomyosarcoma ${ }^{12-13}$. Recent studies have also found that monoclonal antibodies targeting mTOR signaling pathways, vascular endothelial growth factor receptors (VEGF) and insulin-like growth factor receptors have a certain effect on some refractory leiomyosarcoma patients. Pazopanib is a novel oral VEGF-2 inhibitor that also targets both platelet-derived growth factor receptors and stem cell factor receptors (c-kit). The clinical study showed that pazopanib was effective in patients with leiomyosarcoma, synovial sarcoma and other types of soft tissue sarcoma, with a median PFS of 4.6 months (control group 1.6 months) and a OS of 12.5 months (control group 10.7 months) ${ }^{14}$. Anlotinib is a novel small molecule multi-target tyrosine kinase inhibitor developed by China. Similar to pazopanib, it can inhibit many targets, such as VEGFR PDGFR C-Kit ${ }^{15}$, and has anti-tumor angiogenesis and inhibition of tumor growth without significant toxicity ${ }^{16}$. Clinical trials had shown that enlotinib is safe and effective in the treatment of soft tissue sarcoma after failure of standard chemotherapy ${ }^{17}$. After radical operation, no other treatment was performed. Until now, the patient has been followed up for 78 months and no tumor recurrence, which may be related to the earlier tumor staging.

\section{Conclusions}

Retroperitoneal leiomyosarcoma is rare and with few reports in the literature. Its clinical features are not specific. Because of its atypical clinical manifestations, particular attention should be paid to avoid misdiagnosis and missed diagnosis. Because this is a rare type of tumor and lack of clinical research on large samples, the treatment method is still in the exploratory stage. Surgical resection is the preferred treatment method, and multiple treatments such as radiotherapy and chemotherapy may help prolong the survival time of patients. 


\section{Declarations}

\section{Acknowledgments}

The authors would like to thank our patient for sharing his presentation for this manuscript.

\section{Authors' contributions}

All authors contributed to the study conception and design. Material preparation and data collection were performed by Bing Zhang, Mingxia Zhang, Wenjuan Yang and Yiming Qi. The first draft of the manuscript was written by Yunfu Shi, Xiufang Hong and all authors commented on previous versions of the manuscript. All authors read and approved the final manuscript.

\section{Funding}

This study was supported by the Zhejiang Province Famous Traditional Chinese Medicine Experts Inheritance Studio Construction Project (GZS2017001), the Traditional Chinese Medicine Science and Technology Project of Zhejiang Province(2017ZB013, 2018ZA005), and the Medical Science and Technology Project of Zhejiang Province(2018KY198).

\section{Availability of data and materials}

The data are available from the corresponding author on reasonable request.

\section{Ethics approval and consent to participate}

Not applicable.

\section{Consent for publication}

Written informed consent was obtained from the patient for the publication of this case report.

\section{Competing interests}

The authors declare that they have no competing interests.

\section{Author details}

${ }^{1}$ Department of Oncology, Tongde Hospital of Zhejiang Province, Hangzhou, Zhejiang Province, People's Republic of China. ${ }^{2}$ The First Clinical Medical College, Zhejiang Chinese Medical University, Hangzhou, Zhejiang Province, People's Republic of China. ${ }^{3}$ Department of General Surgery, Tongde Hospital of Zhejiang Province, Hangzhou, Zhejiang Province, People's Republic of China. ${ }^{4}$ Department of Geriatrics, Zhejiang Hospital, Hangzhou, Zhejiang Province, People's Republic of China.

\section{References}


1. Brennan MF, Antonescu CR, Moraco N, et al. Lessons learned from the study of 10,000 patients with soft tissue sarcoma. Ann Surg. 2014; 260: 416-21.

2. Pham V, Henderson-Jackson E, Doepker MP, et al. Practical Issues for Retroperitoneal Sarcoma. Cancer Control. 2016; 23: 249-64.

3. Toulmonde M, Bonvalot S, Méeus $\mathrm{P}$, et al. Retroperitoneal sarcomas: patterns of care at diagnosis, prognostic factors and focus on main histological subtypes: a multicenter analysis of the French Sarcoma Group. Ann Oncol. 2014; 25: 735-42.

4. Smith HG, Panchalingam D, Hannay JA, et al. Outcome following resection of retroperitoneal sarcoma. Br J Surg. 2015; 102: 1698-709.

5. Mankin HJ, Casas-Ganem J, Kim JI, et al. Leiomyosarcoma of somatic soft tissues. Clin Orthop Relat Res. 2004: 225-31.

6. O'Sullivan PJ, Harris AC, Munk PL. Radiological imaging features of non-uterine leiomyosarcoma. Br J Radiol. 2008; 81: 73-81.

7. Hartmann DS, Hayes WS, Choyke PL, et al. From the archives of the AFIP:leiomyosarcoma of the retroperitoneum and inferior vena cava: radiologic-pathologic correlation. Radiographics. 1992; 12: 1203-20.

8. Weiss SW. Smooth muscle tumors of soft tissue. Adv Anat Pathol. 2002; 9: 351-9.

9. Lang H, Nussbaum KT, Kaudel P, et al. Hepatic metastases from leiomyosarcoma: a single-center experience with 34 liver resections during a 15-year period. Ann Surg. 2000; 231: 500-5.

10. Eilber FC, Eilber KS, Eilber FR. Retroperitoneal sarcomas. Curr Treat Options Oncol. 2000; 1: 274-8.

11. Sampath S, Hitchcock YJ, Shrieve DC, et al. Radiotherapy and extent of surgical resection in retroperitoneal soft-tissue sarcoma: multi-institutional analysis of 261 patients. J Surg Oncol. 2010; 101: 345-50.

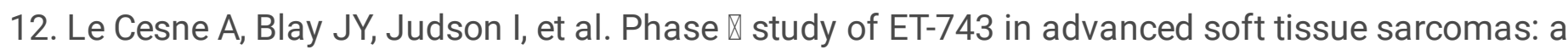
European Organisation for the Research and Treatment of Cancer (EORTC) soft tissue and bone sarcoma group trial. J Clin Oncol. 2005; 23: 576-84.

13. Demetri GD, Chawla SP, von Mehren M, et al. Efficacy and safety of trabectedin in patients with advanced or metastatic liposarcoma or leiomyosarcoma after failure of prior anthracyclines and ifosfamide: results of a randomized phase $\mathbb{\nabla}$ study of two different schedules. J Clin Oncol. 2009; 27: 4188-96.

14. Van der Graaf WT, Blay JY, Chawla SP, et al. Pazopanib for metastatic soft-tissue sarcoma(PALETTE): a randomised, double-blind, placebo-controlled phase 3 trial. Lancet. 2012; 379: 1879-86.

15. Beedie SL, Mahony C, Walker HM, et al. Shared mechanism of teratogenicity of anti-angiogenic drugs identified in the chicken embryo model. Sci Rep. 2016; 6: 30038.

16. Sun Y, Niu W, Du F, et al. Safety, pharmacokinetics, and antitumor properties of anlotinib, an oral multi-target tyrosine kinase inhibitor, in patients with advanced refractory solid tumors. J Hematol 
Oncol. 2016; 9: 105.

17. Chi Y, Fang Z, Hong X, et al. Safety and efficacy of anlotinib, a multikinase angiogenesis inhibitor, in patients with refractory metastatic soft-tissue sarcoma. Clin Cancer Res. 2018; 24: 5233-8.

\section{Figures}
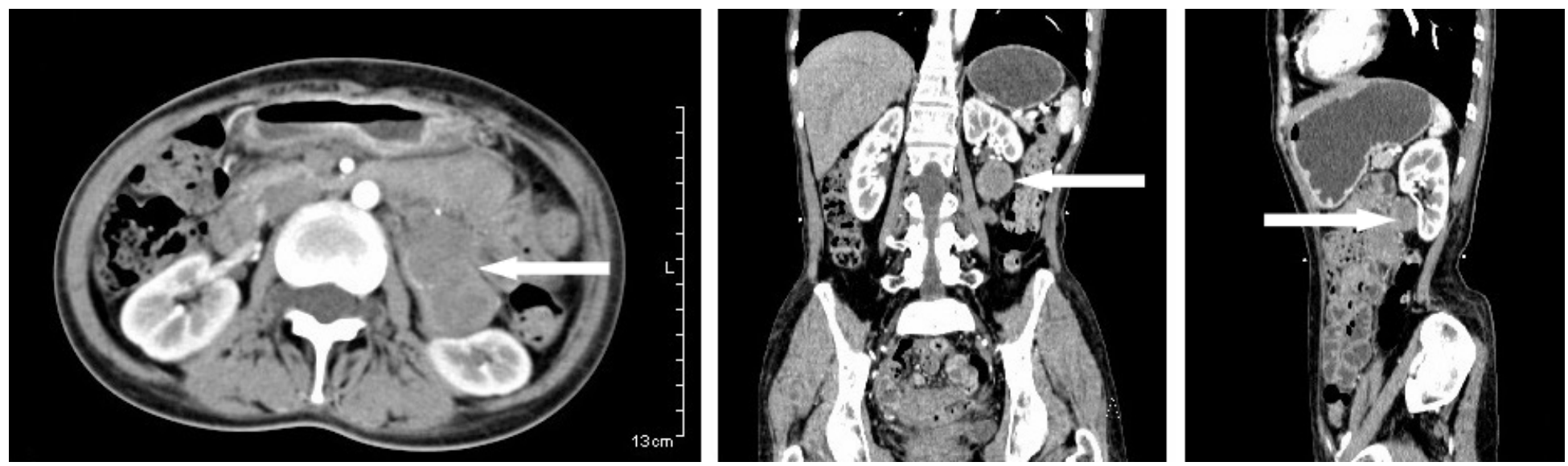

Figure 1

Abdominal computed tomography scan on 29 April 2014 showed that the tumor was located on upper left retroperitoneum.
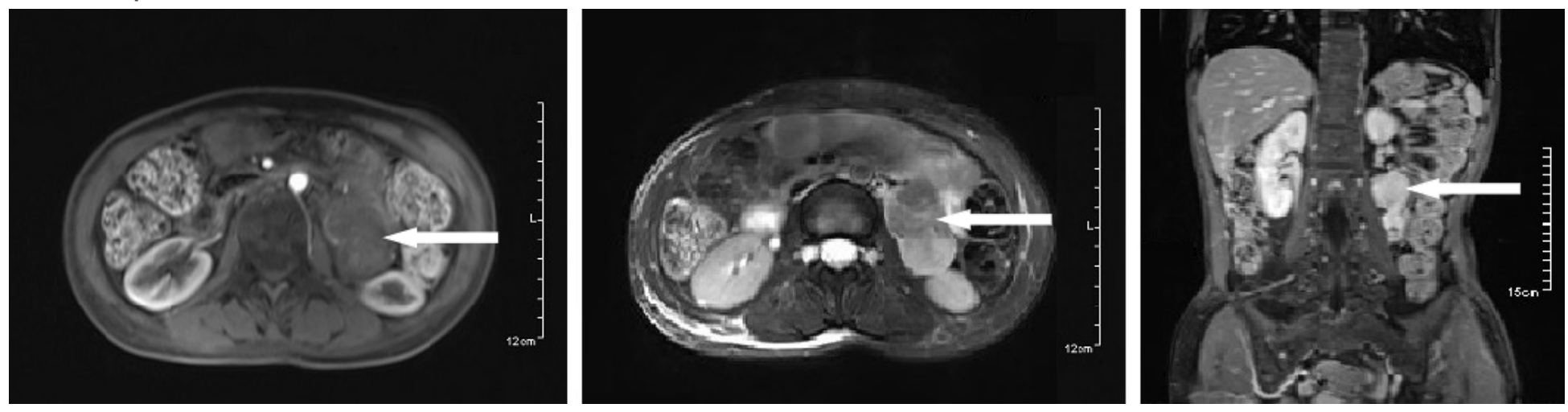

Figure 2

Abdominal magnetic resonance imaging on 6 May 2014 showed that the tumor was located on the left side of psoas major muscle. 

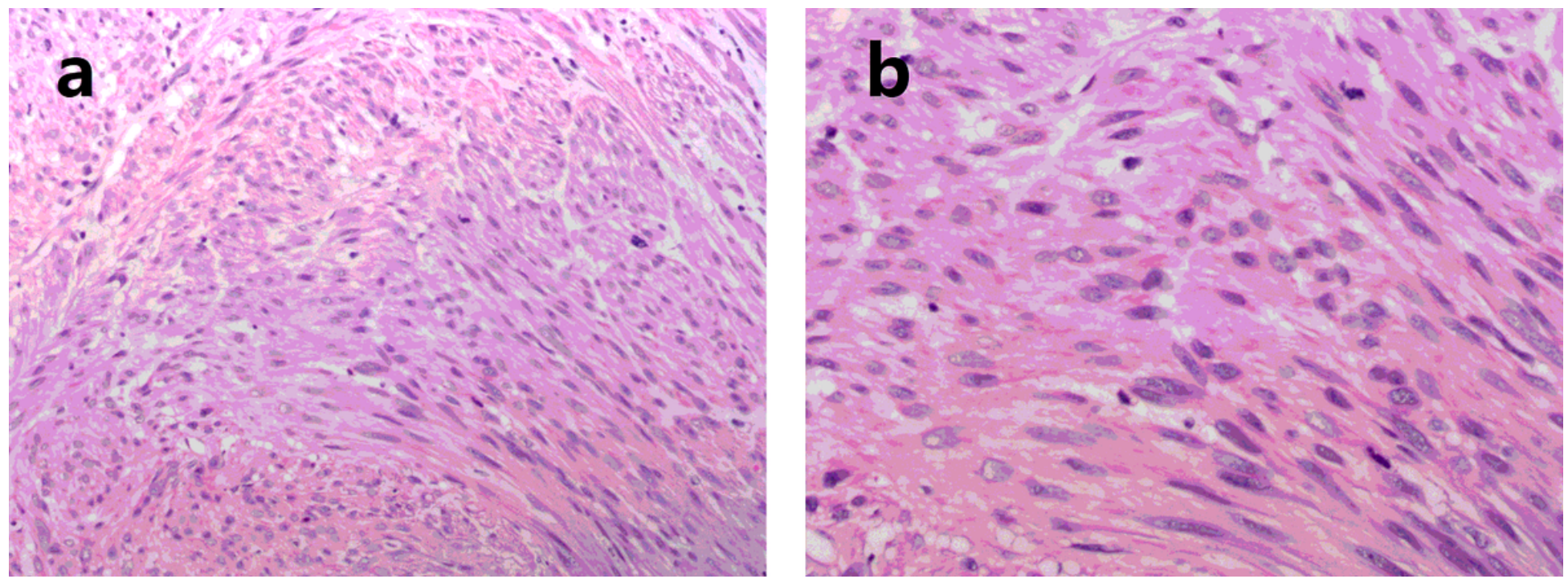

Figure 3

Representative pictures of hematoxylin and eosin staining of tumor tissue. (a) $\times 100$. (b) $\times 200$.
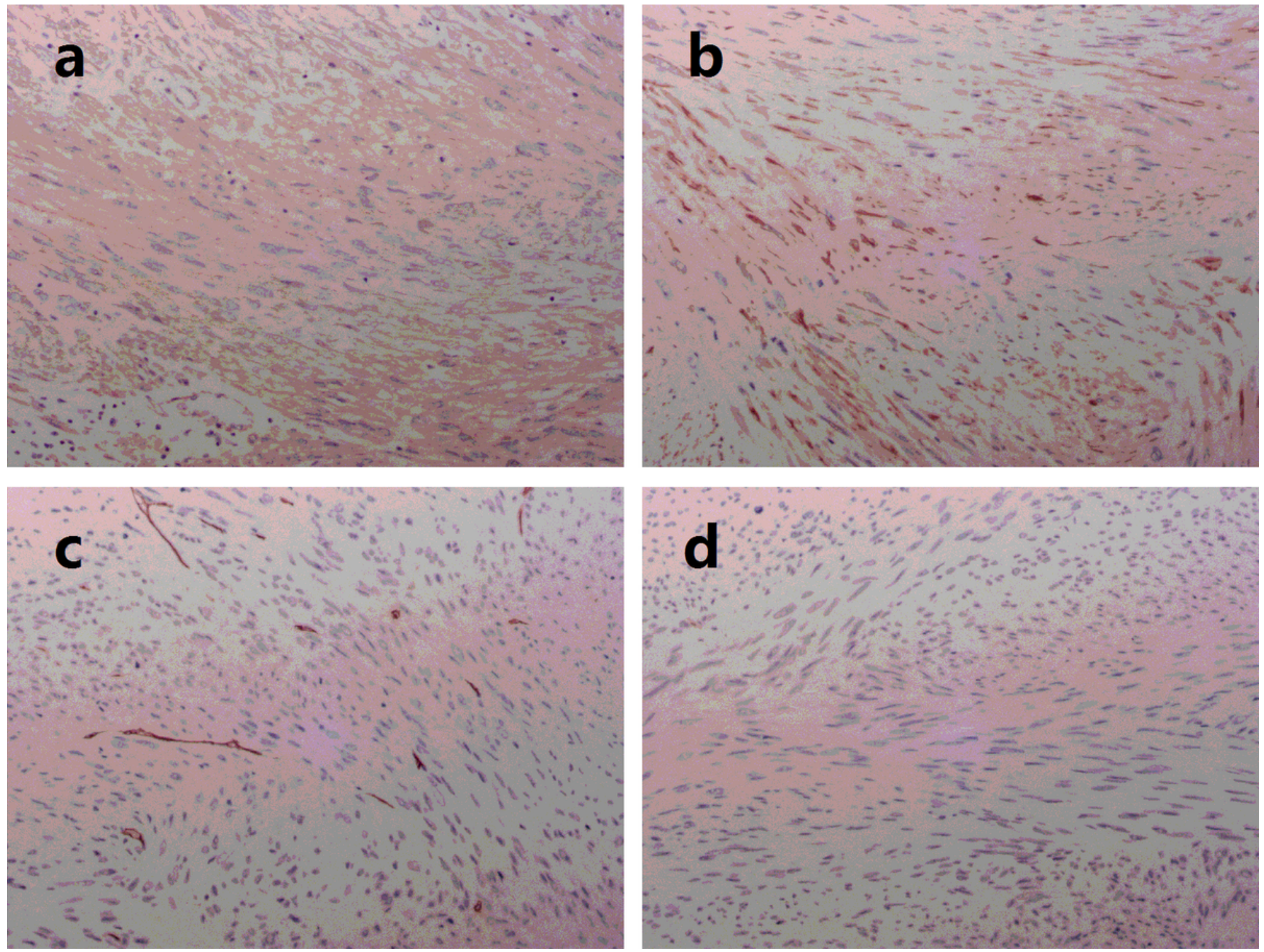

Figure 4 
Representative pictures of immunohistochemistry of tumor tissue. (a) SMA(+). (b) Des(+). (c) CD34(-). (d) CD117(-). 